\title{
Focus on MALDI imaging
}

\section{Olivier Laprévote}

Published online: 27 May 2011

(C) Springer-Verlag 2011

Matrix-assisted laser desorption/ionization (MALDI) emerged in the late 1980s concomitantly with electrospray ionization and contributed substantially to the promotion of mass spectrometry as an invaluable tool for analysis of biological macromolecules. Because MALDI is based on the production of ions from solid-phase samples deposited onto a conducting surface, some researchers attempted to analyse directly biological materials without any preliminary extraction step. MALDI analysis of bacterial cultures as early as the mid-1990s illustrates this approach, the objective of these studies being to characterize and identify microorganisms on the basis of a set of characteristic peaks present in their mass spectra. Identification of bacteria by MALDI mass spectrometry is now a routine method for clinical use.

The ability of MALDI mass spectrometry to obtain spectra on well-defined surfaces with a focus of approximately 50-100 $\mu \mathrm{m}$ should also enable linking of the histological characteristics of biological tissue with local molecular composition. The driving idea was that tissue heterogeneity could be correlated with a difference in the mass spectral peak patterns. MALDI "profiling" of tissue sections was then proposed by the Todd and Caprioli groups at the end of the 1990s to differentiate, on a molecular basis, healthy or pathological areas present within the same tissue section. Soon after, the Caprioli team proposed a method for systematic profiling in which mass spectra were recorded at regularly

Published in the special issue MALDI Imaging with Guest Editor Olivier Laprévote

O. Laprévote $(\bowtie)$

Faculté des Sciences Pharmaceutiques,

Laboratory of Chemistry and Analytical and Cellular

Toxicology, Université Paris Descartes,

4 avenue de l'Observatoire,

75270, Paris cedex 06, France

e-mail: olivier.laprevote@parisdescartes.fr spaced points on a biological surface, leading to a global vision of the molecular composition of the sample. By translating the relative intensity values of the ion peaks into a colour code, differences in local abundances in the $x-y$ plane of the sample were revealed as contrast changes in the generated images. Immediately, MALDI mass spectrometry imaging (MALDI-MSI) seemed to be a unique method to record simultaneously as many images as ion peaks present on the mass spectra and without any a priori information about the nature of the detected molecules. It is therefore logical that MALDI imaging has initially been developed for research on biomarkers of pathologies, in a context in which the "omics" methods, in particular proteomics, have experienced explosive growth.

If MALDI imaging was originally devoted to protein analysis only, this approach quickly met its limits in this area. Measuring the protein ions at low or moderate mass resolution is, indeed, not sufficient for their straightforward identification. In this area, MALDI imaging seemed more a complementary step to conventional proteomic analysis rather than an alternative method. In this way, MALDI imaging can provide precious indications of in situ protein-expression changes, making this technique attractive as a future diagnostic tool in biomedical research (Hanrieder et al., in this special issue).

Recent years have seen a growing number of methodological developments and applications of MALDI imaging in the field of "small molecules". For such compounds, accurate mass measurements, associated or not with tandem mass spectrometry (MS-MS), are often sufficient for assignment of the structures of the detected ions. Illustrating this evolution, the analysis of lipids is now a major field of application of MALDI imaging (Fernandez et al., Garrett et al., and Hart et al., in this special issue). 
Nevertheless, many practical problems have arisen since the origin of MALDI-MSI and remain detrimental to its popularization. Sample treatment before the analysis and matrix-deposition processes has been greatly facilitated by commercially available robotic technology. Notwithstanding, the chemistry associated with ionization/desorption of the analyte from a biological environment remains an inherent problem of this approach and the development of MALDI matrices promoting detection of specific species is an important part of expected improvements of the technique (Cerutti et al., in this special issue).

Similarly, the issue of the relevance of the mass spectrometric profiles obtained from biological surfaces in terms of semi-quantitative measurement and of their possible use for the absolute quantification of diagnostic molecules has been raised. Heterogeneity of the biological material can result in heterogeneity of the ionization efficiencies for many compounds. False images with regard to real concentration variations could then be obtained. These matrix effects have to be further studied and better understood in order to implement new methods for limiting the occurrence of such artefacts.

Today, MALDI imaging can be performed on most conventional or tandem mass spectrometers, and dataprocessing software is available. However, extraction of relevant information from the huge amount of data generated by an imaging experiment remains challenging (Deininger et al., Bonnel et al., and Hayasaka et al., in this special issue). MALDI imaging is also an experimentally tricky method and its routine use, for example for clinical applications, seems limited. However, its future use in biomarker discovery and as a clinical diagnostic tool is very promising, assuming appropriate original and efficient strategies are developed (Cazares et al., in this special issue). More generally, the contribution of MALDI imaging in biology is indisputable. In the field of lipids, for example, no other imaging technique is able to locate structurally well-defined molecules in a biological tissue (Marsching et al., in this special issue). Similarly, structural information derived from MS and MS-MS data enables differentiation of the locations of drugs and of their metabolites, whereas these are merged by autoradiography techniques. Finally, efforts devoted to increasing both spatial and mass resolution have led to remarkable performance (Römpp et al., in this special issue).

The dynamism of the scientific community involved in this area, the enlargement of its scope to other disciplines in chemistry and physics (Weidner et al., in this special issue), the constant improvement of the instrumentation, the simplification of experimental procedures, and the needs expressed by the biology and medicine communities make MALDI imaging one of the most promising approaches of mass spectrometry and, in general, of analytical and bioanalytical chemistry.

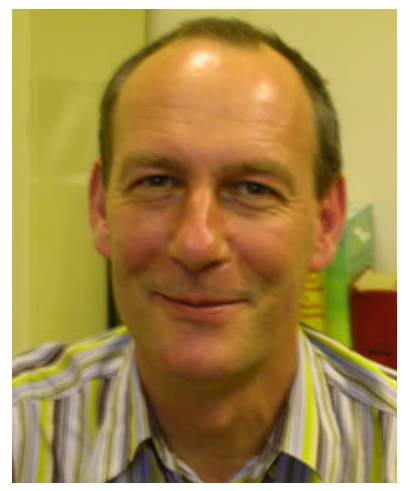

Olivier Laprévote is currently Professor of Toxicology at the University Paris Descartes (Faculty of Pharmacy) and at Lariboisière Hospital in Paris. He was Group Leader of the Mass Spectrometry Laboratory of the Institute for Natural Compounds Chemistry at Gif-sur-Yvette (France) until 2008. His scientific interests lie in the development of original mass spectrometric methods for characterization of natural molecules in complex biological matrices. Among his past research activities, mass spectrometry imaging took a prominent part, with a particular interest being in biomedical applications. O. Laprévote is a member of the Advisory Board of Analytical and Bioanalytical Chemistry. 